\title{
An Agent Oriented Approach of Requirement Engineering in Developing a Data Ware Houses for Banking System
}

Sandeep Mathur
Girish Sharma

A K Soni

\begin{abstract}
Most of the data ware house project fails to meet the business requirements and business goals because of the improper requirement engineering phase. The chaos all through the development of requirements evolves due to disparity between users and developers resulting in project devastations and terminations. Building a data warehouse is a very challenging task. Data warehouse quality depends on the quality of its requirement engineering models. Agent orientation is emerging as a unique paradigm for constructing Data ware house. Agent oriented systems are expected to be more powerful, more flexible, and more robust than conventional software systems. In this paper the detail discussion of agent oriented methodology used in early as well as late requirement elicitation. The proposed approach is illustrated through an case study of the general banking system for which Data Ware house is to be built to support decisional goals.
\end{abstract}

Keywords - Data ware house (DWH), Requirement elicitation, agents, Goal decision information model (GDI), AGDI.

\section{INTRODUCTION}

Critical business decisions depend upon the availability of proper strategic information in the enterprise [1], [5], [7]. Data Warehouse (DW) systems are used by decision makers to analyze. DW acknowledged as one of the most complex information system modules and its design and maintenance is characterized by several complexity factors.

The solution of the aforesaid problem is data ware house. The data warehouse is primarily used for the decisional purposes and supports on-line analytical processing. The data in data warehouse is historical in nature available in very huge amount. Because of these basic requirements of a data warehouse system, the development of a data warehouse system is also different from the development of a conventional operational system. Therefore the data warehouse design process has not been supported by a formal requirements analysis method though there are some approaches for requirements gathering. Thus requirements engineering for the data warehouse aims to identifying the information needs of the decision-makers. In recent years, requirements engineering for DW has acquired importance. [7], [13], [17]. A relationship of the Data Warehouse to the organizational context is established at the requirements level. The requirements Engineering task has been divided into two phases: early requirements engineering phase and late requirements engineering phase [4], [23]. The early phase of requirements engineering activities include to consider how the intended system would meet organizational goals, why the system is needed, The emphasis here is on understanding the "whys" that underlies system requirements, rather than on the precise and detailed specification of "what" the system should do. The late requirement analysis describes the developing system within its operational environment along with its function and properties. Now this phase specifies what the system will do and how it will be done.

\section{Overview of Goal decision Information model for DWH Requirement Engineering}

Goal- decision model covers both the analysis of initial requirements and the specification of these requirements in terms of a conceptual schema. Goal-oriented requirements analysis starts with a list of stakeholders and their high-level goals, which are refined and interrelated to produce a goal model in which only major stakeholders or decision making users are involved in requirement elicitation process [12] .

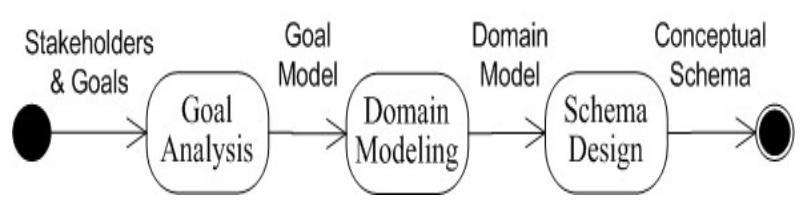

Fig 1: GDI model

Goal-oriented schema design is further divided into two Stages [17]: the modeling of the application domain which describes the necessary understanding of a part of the real world, and facilitates the communication of domain knowledge between developers, end-users and other stakeholders and a conceptual schema, that represents the semantics of the actual data in the proposed database; its design focuses on issues that are specific to the conceptual content and organization of the data. 
The GDI [14], [19] model starts with the determination of goal of the organization with the help of decision makers by assuming that only decision maker are the stakeholders responsible for decision making activities in the organization. The other stakeholders and their dependencies for achieving the goals of the organization are also important to be modeled. For this GDI model does not have any notion to model stakeholder explicitly

\section{Agent Goal Decision Information Model}

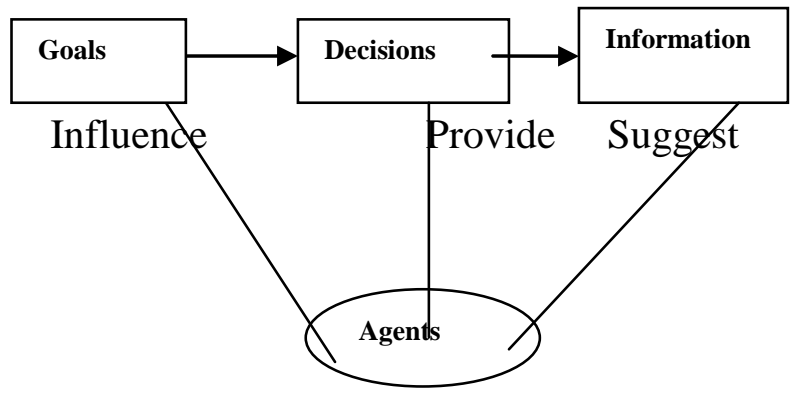

Fig 2: Snap short of Agent Goal Information model

The notion agent represents each and every user of the system. They can be stock holders, decision makers, information providers etc. The organizational goals can be achieved by combining the individuals goals of each stakeholders which can be in turn depends on each other. The Agent may depend on another agent for goals to be achieved, decisions to be suggested \& information to be provided. These dependencies among agents are called goal, decision \& information dependencies respectively. The extended GDI model is called an AGDI model [8], [9]. AGDI model is used to support organization modelling and goal modelling activities. AGDI uses the following concepts for requirement modelling

Agents- position of an agent can be considered as actors of use case diagram. It represents the various users, stakeholders, decision makers and users of the organization. [8] Defines various different types of roles that termed as actors. The agent can be internal, external depending upon the working of the agents' w.r.t. to system. Agents can be either simple agents or complex agents.

Agents \& goals - Goal are the objectives of agents to be achieve by the organization. These goals are the prime driving force in requirement elicitation for the developing DWH. Goals can be simple or complex that can be subdivided into simple goals. These goals may have two or more actors' dependencies to achieving a particular goal.

Agent, decision \& Information - once the goals have been decided it needs a decision and information on which decision has to be made to achieve the goals. These decisions for achievement of goals will be suggested by to agents thought repeated interactions. The DWH itself as an agent provide information to support these decisions.

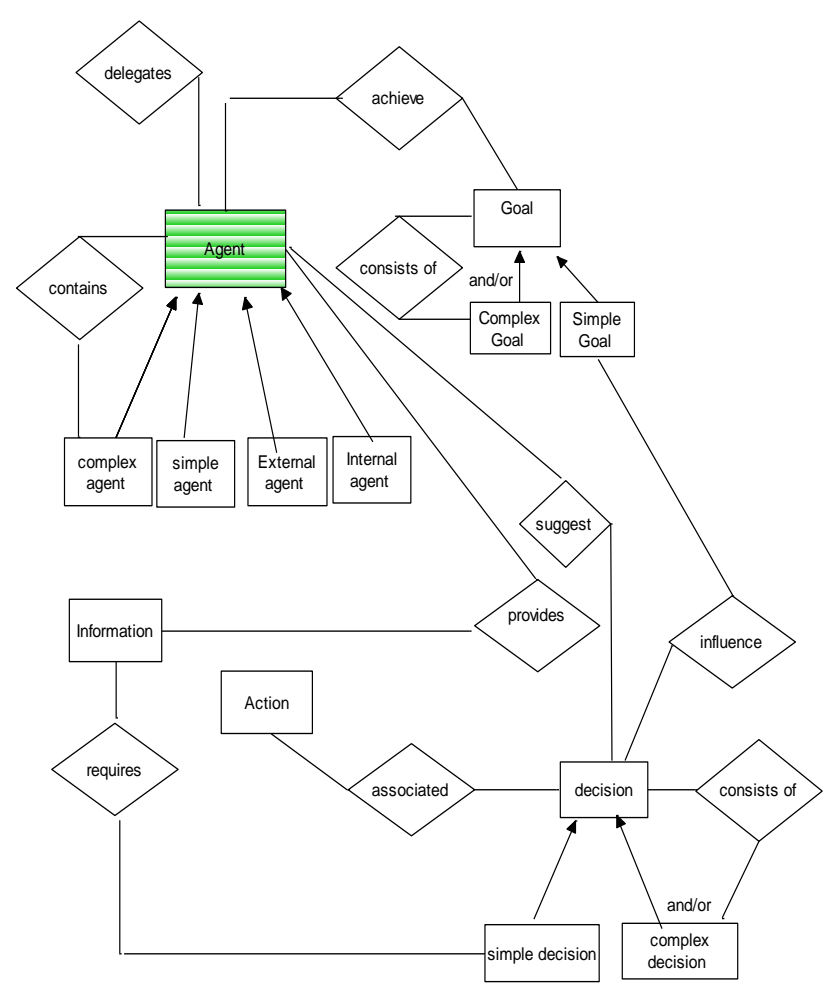

Fig 3: AGDI model

In AGDI the early phase requirement analysis can achieve by Organization model which provides agents with their goal dependencies and Goal model which provides the relevant decisions to achieve the previously defined goals whereas late requirement analysis is achieved by Decision model that provide quality information to support the decisions made earlier.

To illustrate this approach we take an example of general banking system where each and every agent influences the organization, goal \& decision models.

\section{A. Organization modelling}

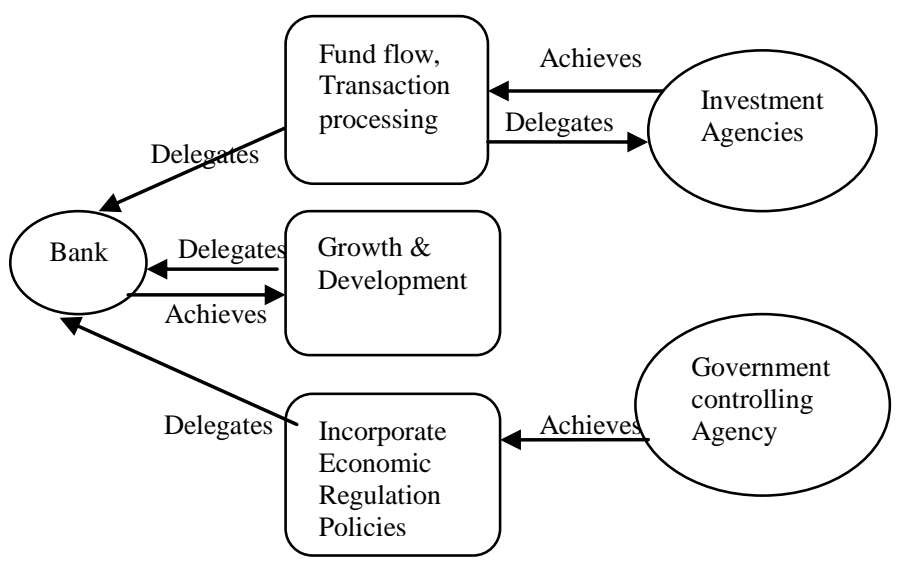

Fig 4: Organization model 
In the organization modeling, we identify the various stakeholders as agents and their expectation as goals of the agent. Here in our banking example Government controlling agency such RBI in India and investment agencies are external agents whereas bank itself is a complex agent - customer, staff \& higher management.

Bank has three goals to achieve:

a) Fund flow - Perform money transfer activities to get cash in hand for investments. Now this goal can be achieved by the investment agencies to invest the bank cash and bank itself is delegate to achieve this goal as only bank headquarters have the authority of investment. This cash flow is also achieves by routine bank financial activities performed by the bank staff.

b) Incorporate Economic Regulation Policies - various economic policies and rules have been incorporated by government as per the country wide prospective. Government financial agencies such as Reserve bank of India delegate bank to apply these policies to get benefit for global economy. Bank staff and management works as per these regulations

c) Growth \& Development - This is the main objective of any financial organization. Our focus is on this goal.

We explore this particular objective in detail while discussing the sub goals and decisions with information requirement to achieve this goal.

The bank itself and other external agents such as investment agencies are delegates to achieve this goal. Now let us consider the bank's Designation hierarchy:

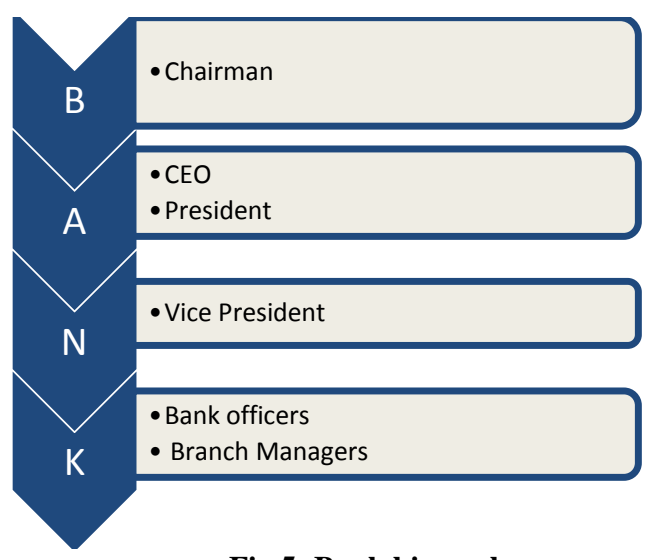

Fig 5: Bank hierarchy

These will work as internal agent for the banking system. These agents are responsible to accomplish the goals so these agents' delegates to achieve the goals and its sub goals. These agents will have roles and positions to play in achieving the goals of the bank. It must be in notice that many a time more than one sub goals are delegated to one agent. This will show the agent goal dependency in the banking system.

Based on the above hierarchy and goal dependency between the actors, the organization model can be simplified and reconstructed as shown in fig 6 . As chairman is the most influential and powerful person on post, chairman is delegates to achieve growth and development goal. Growth and development can be achieved in terms of economic growth, infrastructural growth and HR growth. Therefore these three are the sub goals of the main goal. Further these sub goals have been more classified into simple sub goals. Such as to get economical growth bank need to gain maximum profit and more returns on the investments. Next from the bank designation hierarchy president is delegate to achieve these 2 simple sub goals as president is allowed to invest the bank's cash into various agencies. Similarly hire efficient staff will have simple sub goal staff welfare policies whereas infrastructural growth can be achieved by opening more and more new branches to involve more and more customer while development in the bank's organization. Chief executing officer of any bank has to deal with these extension and development decisions therefore CEO is delegates to achieve these 2 simple sub goals. This simplified organization model haves the simple goals to be achieve by various agents

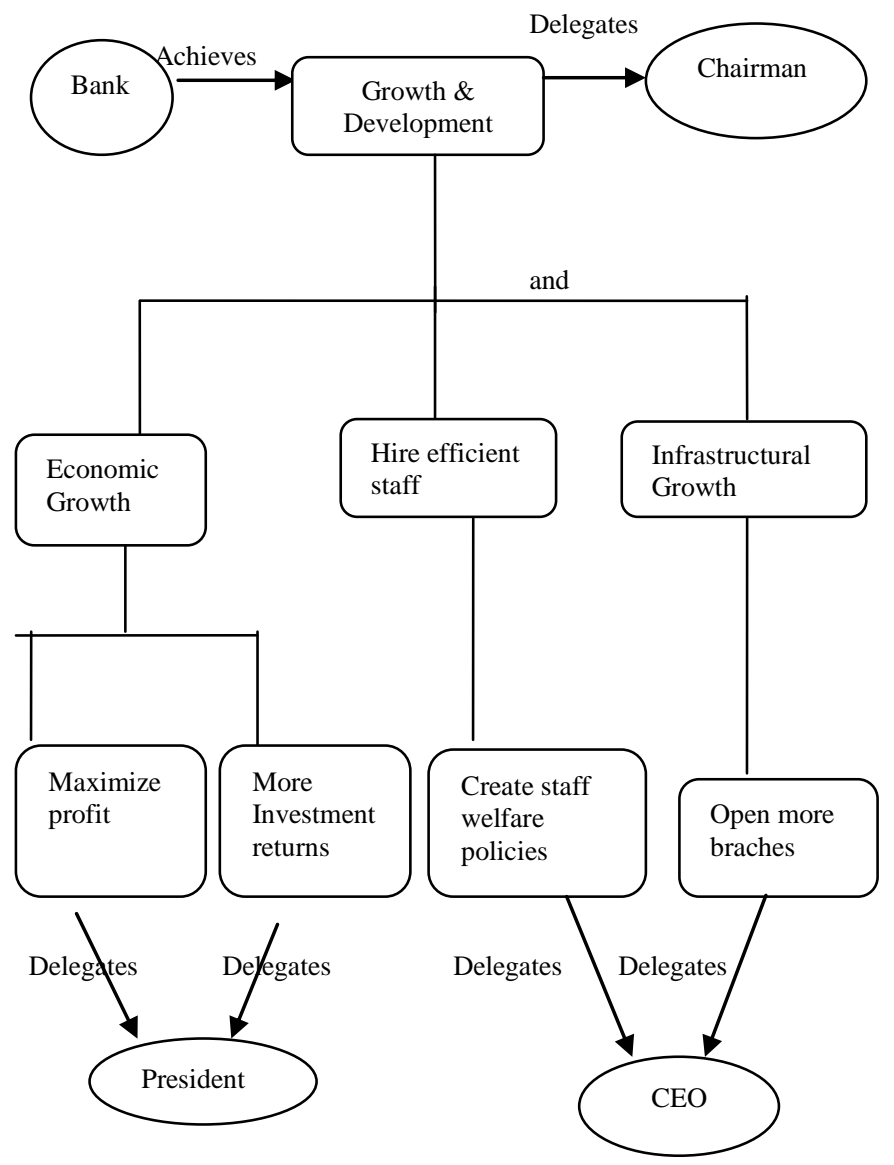

Fig 6: Simplified organization model

\section{B. Goal modeling}

In the goal modeling agents will suggest various decisions that will have to taken to achieve the goals mentioned in the organization model. Considering our main objective discussion and keeping the fig 6 , we will continue with maximize profit goal. As shown in fig 
agent president is delegate to achieve this goal by suggesting simple and complex decision as shown in fig

7. It shows the following decisions to be made:

- $\quad$ Reduce expenses

- Increase earning>Attract more customers

- Loan interest

Here decision 2, 3 are complex decisions which is further broken into one simple sub decision. To get maximum profit bank need to reduce its expense this will be explore in future. Now these all simple decisions and manager is delegated to take them.

It is also a fact that Profit gaining though internal agent as a bank is a combined efforts by each bank branch so branch head known as branch manager is delegated to achieve this goal by taking above mentioned simple decisions.

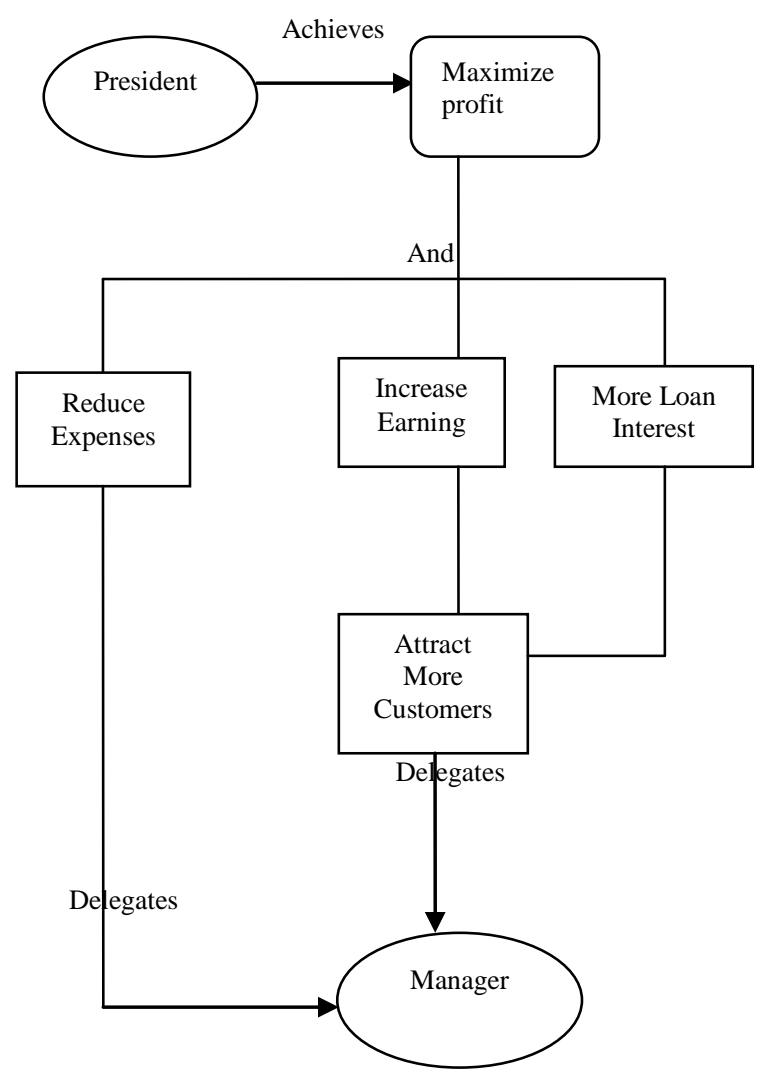

Fig 7: Goal model

\section{Decision Modeling}

In the decision modeling, agents are needed information to support the indentified simple decisions during the goal modeling. Here in our example to support the simple decision "attach more customer" made in goal modeling requires following set of information by the agent manager:

a. Popular customer friendly policies- pick the best bank policies and procedures, popular in the existing customers for user friendly banking to get attract more depositors and customers. In this way more funds are available in the bank for loan and investments.

b. List of customers using bank facilities- list out the bank customers who are using bank credit/debit cards, ATM cards and do nonfinancial transactions such as mini statements, balance enquiry etc. As bank charges fees to provide these services this will ultimately increases the bank earnings. This list also provides the list of customers who are not currently using any of the bank beneficial schemes such as sms alerts, insurance policies of bank etc. and agent branch manager can motivates them to avail such a bank offers. In this way bank get more customers and hence get more earning.

c. Records of loan interested customers - list the customers who are interested to availing any type of loan from the bank as the bank majorly earn profit from the difference of interest rate paid to the depositors of saving accounts and the loan repayment interest accrued by the bank from the borrower. For example say bank will give $8 \%$ interest rate on saving bank accounts whereas charges $12 \%$ interest rate on loan accounts. So $12-8=4 \%$ is the bank profit. Therefore increase of the loan amount will increase the bank earnings. This will be subject of availability and eligibility. Hence personal and financial information of loan interested customers is also required by the branch manager. 


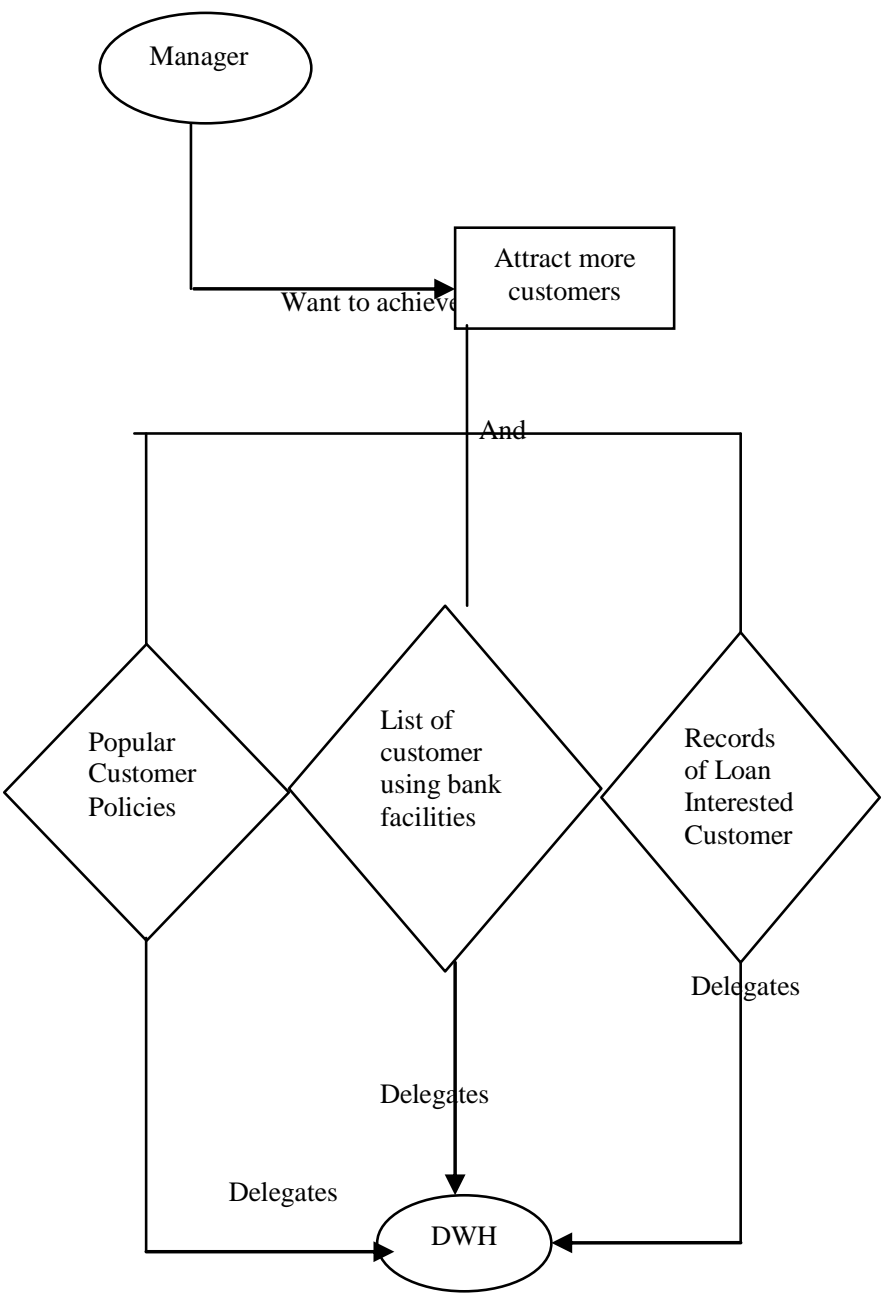

Fig 8: Decision model

To provide these set of information there is a need of an agent which can manage this kind of historical information in an effective way. In this case data ware house is a viable solution, which will work as decisional system for the organization. Similarly to support all suggested decisions the required information may be indentified though set of interactions among agents. This set of information will be kept in DWH. The set of models produced during the early and late requirement analysis will kept in DWH.

\section{Conclusion}

The set of decision models produced during decision modeling activities captures the late requirement for a DWH whereas organization and goal models produced captures the early requirements. DWH is itself act as an agent that provide the information support needed for the decisions made at goal modeling. Keeping this fact the requirement elicitation and analysis for the developing DWH is closer to the practical aspect. As it's ultimately the user who will use and get benefits by the DWH therefore requirement elicitation by using this approach by involving each agent of DWH is more suitable than other conventional elicitation techniques. This approach has been discussed by taking general banking system case study.

\section{Future Work}

In the coming future, more options by focusing on the different system agents and various goals of the banking system will be explored, other than growth and development. AGDI can be applicable in other fields such as medical and other domains. Combing of AGDI with troops methodology can extend the accuracy and having more practical approach.

\section{References}

[1] Golfarelli, M. and Rizzi, S, Designing the Data Warehouse: Key Steps and Crucial Issues" in Journal of Computer Science and Information Management, Vol. 2 No.3, 1999.

[2] Gregoriades, A. and Sutcliffe, A. Scenario-based assessment of nonfunctional requirements" Software Engineering, IEEE Transactions on Volume 31, Issue 5, May 2005, Pages: $392-409$.

[3] Paolo Giorgini, Stefano Rizzi, Maddalena Garzetti GRAnD Goal oriented requirement design for dataware house design. Decision Support Systems journal, Elsevier, pp. 4-21, Vol 45 Issue 1, 2008.

[4] Paolo Giorgini \& Brian Henderson-Sellers and Fausto Giunchiglia Tropos:A Agent Oriented methodology Issue 2005

[5] Hüsemann, B., Lechtenbörger, J., Vossen, G.: Conceptual Data Warehouse Design. In: Proceedings of the International Workshop on Design and Management of Data Warehouses DMDW 2000 Stockholm, Sweden, pages: 5-6.

[6] Prakash, N., Gosain, A., An approach to engineering the requirements of data warehouses, Springer-Veralag, Requirements Eng. Journal, 2007.

[7] Naveen Prakash, Deepika Prakash, and Y.K. Sharma Towards Better Fitting Data Warehouse Systems, A. Persson and J. Stirna, International federation for information Processing 2009, Pages: 130- 144.

[8] Yogesh Singh, Anjana Gosain, Manoj Kumar from Early Requirements to Late Requirements Modeling for a Data Warehouse. Fifth International Joint Conference on INC, IMS and IDC, 2009.

[9] Wilmann and Leon Damien Sterling, Guiding agentoriented requirements elicitation: HOMER, University of Melbourne Proceedings of the Fifth International Conference on Quality Software (QSIC'05), 2005.

[10] Matteo Golfarelli's survey on User

[11] Requirements to Conceptual Design in Data Warehouse Design, 2008.

[12] Ines Gram and Camellie Salinesi. A Requirement-driven Approach for Designing Data Requirement Engineering: Foundations for Software Quality (REFSQ 06), Luxembourg 2006. 
[13] Prakash, D., Goal Oriented Requirements Engineering for Data Warehousing, M.Tech. thesis, IIIT Bangalore 2009.

[14] Robert M. Bruckner, Beate List, Josef Schiefer. Developing requirements for data warehouse systems with use case, seventh American conference on Informational systems 2001.

[15] Saroop S.; Kumar M, Comparison of Data Warehouse Design Approaches from User Requirement to Conceptual Model: A Survey. In Communication Systems and Network Technologies (CSNT),2011 International Conference on pages: $308-312$.

[16] Vibha Gaur, Anuja Soni, Punam Bedi: An Agent-Oriented Approach to Requirements Engineering in IEEE 2nd International Advance Computing Conference (IACC), 2010 On pages: $449-454$.

[17] Umashakar Sharma Anjana Gosain Navin Rajpal Comparison of different Methodologies for dataware house Development International Journal of Research in IT, Management and Engineering IJRIME 2011, Volume1 Issue3 ISSN: 2249- 1619, Pages: 354-364.

[18] Lei Jiang, Thodoros Topaloglou, Alex Borgida, John Mylopoulos Goal-Oriented Conceptual Database Design 15th IEEE International Requirements Engineering Conference 2007.
[19] Robert Grover Little Jr. and Michael Lucas Gibson Perceived Influences on Implementing Data Warehousing, IEEE Transactions on Software Engineering, Vol. 29, No. 4, published in 2004.

[20] Shashank saroop and Manoj kumar, Comparison of data ware house design approaches from user Requirement to conceptual model A Survey. International conference on communication systems and network technologies, 2011, page: 976.

[21] Jesús Pardillo and Jose-Norberto Mazón, using ontologies for the design of data warehouse, International journal of database management system (IJDMS), Vol 3 May 2011.

[22] Winter, R., Strauch, B., "Information Requirements Engineering for Data Warehouse Systems". ACM Symposium on Applied Computing (SAC'04) Nicosia, Cyprus.2004

[23] Rilson, F. and Freire, J., "DWARF: AN Approach for Requirements Definition and Management of Data Warehouse Systems", Proceeding of the $11^{\text {th }}$ IEEE International Requirements Engineering Conference, September 08, 2003, Pages: 1090-1099.

[24] Yu, E.S.K., "Towards modeling and reasoning support for Early phase requirement Engineering", proceedings of IEEE International Symposium on Requirements Engineering, 226-235, 1997 